Available online at

SciVerse ScienceDirect

www.sciencedirect.com
Elsevier Masson France

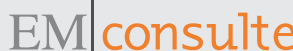

www.em-consulte.com/en

Original article

\title{
The feature of Metabolic Syndrome in HIV naive patients is not the same of those treated: Results from a prospective study
}

\author{
Paolo Bonfanti ${ }^{\mathrm{a}, *}$, Giuseppe Vittorio De Socio ${ }^{\mathrm{b}}$, Elena Ricci ${ }^{\mathrm{c}}$, Andrea Antinori ${ }^{\mathrm{d}}$, Canio Martinelli ${ }^{\mathrm{e}}$, \\ Francesca Vichi ${ }^{f}$, Giovanni Penco ${ }^{g}$, Giordano Madeddu ${ }^{\mathrm{h}}$, Giancarlo Orofino ${ }^{\mathrm{i}}$, Laura Valsecchi ${ }^{\mathrm{c}}$, \\ Stefano Rusconi ${ }^{\mathrm{j}}$, Barbara Menzaghi ${ }^{\mathrm{k}}$, Daria Pocaterra ${ }^{\mathrm{c}}$, Tiziana Quirino ${ }^{\mathrm{k}}$
}

a Department of Infectious Diseases, A. Manzoni Hospital, via dell'Eremo 9, Lecco, Italy

${ }^{\mathrm{b}}$ Department of Infectious Diseases, Santa Maria Hospital, Perugia, Italy

${ }^{\mathrm{c}}$ Department of Infectious Diseases, Luigi Sacco Hospital, Milano, Italy

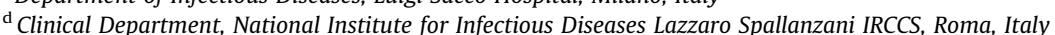

e Department of Infectious Diseases, Careggi Hospital, Firenze, Italy

${ }^{\mathrm{f}}$ Department of Infectious Diseases, Santa Maria Annunziata Hospital, Firenze, Italy

${ }^{\mathrm{g}}$ Department of Infectious Disease, Galliera Hospital, Genova, Italy

${ }^{\mathrm{h}}$ Department of Infectious Diseases, University of Sassari, Sassari, Italy

i Department of Infectious Diseases, Amedeo di Savoia Hospital, Torino, Italy

${ }^{j}$ Clinica Malattie Infettive, University of Milan, Milano, Italy

${ }^{\mathrm{k}}$ Department of Infectious Diseases, Busto Arsizio Hospital, Busto Arsizio, Italy

\section{A R T I C L E I N F O}

\section{Article history:}

Received 4 January 2012

Accepted 25 January 2012

\section{Keywords:}

HIV

Metabolic syndrome

Antiretroviral therapy

Cardiovascular risk

\begin{abstract}
A B S T R A C T
Metabolic Syndrome (MS) is a common disorder combining obesity, dyslipidemia, hypertension, and insulin resistance. Its prevalence among HIV-infected people is still debated. Besides, how antiretroviral therapy and HIV infection per se are related to MS is still unclear. All treatment-naïve patients attending scheduled visits at CISAI group hospitals between January and December 2007 were eligible for the study. Patients without MS at enrolment were followed-up for 3 years or until they developed MS, diagnosed according to the National Cholesterol Education Program (NCEP) definition. The main objective was to assess the 3-years incidence of MS. MS was evaluated for 188 subjects. Out of them, 62 (33.0\%) had started HAART at enrolment, whereas 67 (35.6\%) more started during the observation. 59 (31.4\%) were still treatment-naive at the study end. MS was newly diagnosed in 14 patients. The incidence was 2.60 cases/100 person-years (95\% CI 1.47-4.51), 2.75 (1.11-5.72) among HAART-naïve patients and 2.65 (1.23-5.03) in subjects on HAART. Blood pressure did not change in the study period, whereas in naive patients the HDL level significantly lowered (median -6.0 vs. $4.0, P<0.0001$ ) compared to HAART-treated patients. Triglicerides increased significantly in HAART subjects (median 12.0 vs. 1.0, $P=0.02$ ), as well as blood glucose (median 6.0 vs. $1.0, P=0.01$ ). In our population, the overall MS incidence was low and largely similar in patients who started HAART or remained naive. However, the feature of MS was different in the two groups, suggesting that in untreated and treated patients MS developed through different metabolic pathways.
\end{abstract}

(C) 2012 Elsevier Masson SAS. All rights reserved.

\section{Introduction}

Metabolic Syndrome (MS) is a common and complex disorder combining obesity, dyslipidemia, hypertension, and insulin resistance. In the HIV-positive population, MS is considered an emerging risk factor for overall cardiovascular risk [1-6], though different populations and ethnic groups show prevalence ranging from 7 to 45.5\% [7]. In the clinical setting, diagnosing MS is useful to identify and characterize HIV-infected patients at higher

\footnotetext{
* Corresponding author.

E-mail address: bonfanti.paolo@gmail.com (P. Bonfanti).
}

cardiovascular risk [8]. Nevertheless, how antiretroviral therapy and HIV infection per se are related to MS is still unclear: to date, studies have not provided conclusive results [7].

Recently, we have published the results of the cross-sectional analysis of 292 HAART-naive subjects enrolled in 2007 [9]. Among them, the prevalence of MS was $12.3 \%$, increasing with age and lower education. An interesting though not conclusive result was that treatment-naïve HIV-positive patients in an advanced stage of the disease had a higher prevalence of abnormal levels of triglycerides, HDL cholesterol and blood glucose than those at a less advanced stage. Along the same line, Jacobson et al. [3] found that MS incidence was higher in patients with 6-months HIV RNA

0753-3322/\$ - see front matter @ 2012 Elsevier Masson SAS. All rights reserved. doi:10.1016/j.biopha.2012.01.005 
increase. These findings suggested, therefore, that HIV infection per se was associated to MS.

To add information on the issue, the HERMES project (HIV Exposure and Risk of MS) was conducted by the CISAI group (Italian Coordination for Research into Allergy and HIV Infection), investigating the role of HIV infection and its treatments in the development of MS. This cohort study only included patients who were treatment-naïve at entry. A primary objective of this analysis was to determine the relationship between MS development, HIV infection and HAART.

\section{Methods}

Patients were recruited between January 2007 and December 2007. All treatment-naïve patients, attending the visits scheduled at the start of the study in the CISAI centers, were eligible if they agreed to enter this observational study. Patients were interviewed by a physician, and all laboratory tests were performed at the center of origin, using blood samples taken after 8-12 h fasting. Patients could start antiretroviral treatment after enrolment if indicated.

All participants gave their informed consent to participate in the study, which was approved by the institution's ethics committee.

The following subjects were not eligible: patients who had started antiretroviral therapy; pregnant women; patients with ascites; patients aged less than 18; patients unable to sign the informed consent form.

All centers used a standard data entry form to collect data at enrolment; main demographic details, as sex, age, ethnicity, marital status and years of education were recorded.

Medical history included information on previous occurrences of ischemic heart disease, ictus or transient ischemic attack (TIA), diabetes, dyslipidemia, malignancies in patient or in his/her first degree relatives.

Dieting and drug treatment for diabetes were recorded. Information on previously diagnosed hypertension and/or antihypertensive drug treatment use was collected, as well as blood lipid disorders and related drug treatments. All data were checked with clinical records where available. Information about treatment initiation during the follow-up period was also recorded.

Smoking and alcohol consumption were recorded (detailed description were previously reported [9]) at study entry and end. Information on co-infection with Hepatitis B or C viruses were collected, as well as diagnosis of chronic hepatitis and its cause. The presence of lipodystrophy was also evaluated.

Clinical information regarding HIV infection were collected: risk factor for HIV acquisition, CDC stage, CD4 + cell count and viral load at enrolment, nadir CD4 + cell count, years from first diagnosis of HIV infection.

Patients were followed for 3 years or until MS development, if the diagnose was made before the study end.

\subsection{Definition of Metabolic Syndrome}

Blood pressure was measured with the patient in the sitting position, using a mercury sphygmomanometer and taking the first and fifth Korotkov sounds to indicate systolic and diastolic values, respectively. Waist circumference was measured with the patient standing. Plasma triglycerides, HDL cholesterol, total serum cholesterol, and fasting plasma glucose were measured from a venous blood sample by a radioenzymatic method. MS was diagnosed according to the 2003 Adult Treatment Panel (ATP) III criteria [10] (ie, when at least three of the following alterations were present:

- waist circumference more than $102 \mathrm{~cm}$ in men and more than $88 \mathrm{~cm}$ in women;
- HDL cholesterol level less than $40 \mathrm{mg} / \mathrm{dL}$ in men and less than $50 \mathrm{mg} / \mathrm{dL}$ in women;

- plasma triglyceride value more than or equal to $150 \mathrm{mg} / \mathrm{dL}$;

- fasting plasma glucose value more than or equal to $110 \mathrm{mg} / \mathrm{dL}$ or use of antidiabetic drugs;

- blood pressure value more than or equal to $130 \mathrm{~mm} \mathrm{Hg}$ systolic or more than or equal to $85 \mathrm{~mm} \mathrm{Hg}$ diastolic).

Regardless of the blood pressure or blood glucose values, use of antihypertensive or antidiabetic drugs was taken to indicate the presence of a blood pressure or blood glucose alteration, respectively.

We also calculated the Global Framingham Risk Score (FRS) as a better predictor of subclinical atheromatous lesions [11] at entry and end of the study to compare the variations in treated and untreated subjects; we calculated two FRSs, including age at study entry and age at study end. In order to normalize the FRS for increasing age, at last follow-up we also calculated FRS at last follow-up considering the actual age $(\mathrm{A})$ and the age at enrolment (B).

\subsection{Statistical analysis}

The incidence rate (IR) of MS and the 95\% confidence intervals were calculated as number of new cases per 100 person years, on the whole sample and in subgroups (gender, age at study entry, HAART treatment after enrolment). Confidence intervals (CI) at 95\% for incidence rates are calculated based on Poisson's binomial distribution. Rate ratios (RR) and the 95\% CI were calculated, and logistic regression was used to simultaneously adjust for the potentially confounding effects of selected variables, according to the Cox model. Since in terms of exposure to HAART the risk factor changed over time, patients who started therapy after the enrolment during the study period were considered in the HAART-naïve group when still naïve and in the HAART group thereafter. Patients without MS were considered as the reference group. Age, sex, and variables that resulted being statistically significant from univariate analysis, were included in the regression equations. Variables included in the definition of MS were excluded from the model. A 95\% confidence interval not including 1 along with a $P$ value $<0.05$ was taken as the level of statistical significance.

Changes in blood pressure, waist circumference, HDL cholesterol, triglycerides and blood glucose were calculated as final level minus baseline level. The differences were compared using the analysis of variance. In order to account for potential confounders, a general linear model was used, introducing the confounding variables into the equation. If the variables were not normally distributed, they were rank-transformed before the multivariate analysis. Relationships between continuous variables were also assessed using Pearson's correlation coefficient, if normally distributed, or Spearman's correlation coefficient if not normally distributed.

Logistic regression was used to evaluate the relation between MS and several variables assessed at the same time of MS development or at last visit. We used unconditional multiple logistic regression, with maximum likelihood fitting, including terms for age, sex and other potential confounders. Odds ratios (OR) and the corresponding 95\% CI were calculated.

Heterogeneity $\mathrm{Chi}^{2}$ was used to evaluate the relation between MS criteria and HAART use by drug class (naïve, PI-based and NNRTI-based therapy). To account for potential confounding, we used the Cochran-Mantel-Haenszel method; included variables are listed in the table footnotes. 


\section{Results}

Overall, 292 patients were enrolled. A participating center withdraw from the study after the enrolment period: 26 patients were not followed any more. Out of 266 remaining patients, 31 were excluded from the incidence analysis because they had MS at baseline. Among 235 subjects without MS at baseline, 195 (83\%) were followed-up for 3 years or until MS was diagnosed. Since seven of them lacked at least one criterion for MS diagnosis, we could evaluate MS in 188 subjects.The patients lost to follow-up were mainly males aged less than 50 years (63\%).

\subsection{Metabolic Syndrome incidence}

In the 3-year follow-up period, 14 patients developed MS, three females and 11 males.
The incidence was 2.60 cases/100 person-years (95\% CI 1.47 4.51), $2.35(0.60-6.40)$ in females and $2.80(1.47-4.86)$ in males. Among patients who remained HAART-naïve, the incidence was 2.75 (1.11-5.72), whereas in subjects who started HAART it was $2.65(1.23-5.03)$.

Subjects who entered the study less or equal to 50 years old were less prone to MS: in this group MS incidence was 1.85/100 person-years $(0.86-3.51)$ versus $6.82 / 100$ person-years $(2.49-$ 14.84 ) in people aged more than 50 years. In percentage, MS developed in $11.9 \%$ of subjects who remained naïve over the observation period and in $8 \%$ of patients who started HAART after the enrolment visit.

\subsection{Patients' characteristics}

The patients' characteristics and the risk ratios are shown in Table 1. Gender and risk factor for HIV acquisition were not

Table 1

General characteristics and immunological status of patients evaluated in the HERMES study by Metabolic Syndrome status.

\begin{tabular}{|c|c|c|c|c|c|c|c|c|}
\hline Variable & Total & Without MS & With MS & $P$ & $\mathrm{RR}^{\mathrm{e}}$ & $95 \% \mathrm{Cl}^{\mathrm{e}}$ & $P^{\mathrm{e}}$ & $\begin{array}{l}\text { Reference } \\
\text { variable }\end{array}$ \\
\hline$N$ & 188 & $174(92.5)$ & $14(7.5)$ & - & & & & \\
\hline Age (median, interquartile range) & $38(32-47)$ & $38(32-45)$ & $47(33-53)$ & 0.04 & 3.4 & $1.2-11.0$ & 0.03 & $<50$ \\
\hline$B M I($ mean,$S D)$ & $23.2(3.1)$ & $22.9(2.8)$ & $27.1(3.8)$ & 0.001 & 15.6 & $3.2-69.6$ & 0.001 & $<25$ \\
\hline \multicolumn{9}{|l|}{ Gender ( $n \%)$} \\
\hline M & $142(75.5)$ & $131(75.3)$ & $11(78.6)$ & \multirow[t]{2}{*}{0.83} & 0.9 & \multirow[t]{2}{*}{$0.3-3.6$} & \multirow[t]{2}{*}{0.98} & \multirow[t]{2}{*}{ M } \\
\hline $\mathrm{F}^{\mathrm{a}}$ & $46(24.5)$ & $43(24.7)$ & $3(21.4)$ & & 1 & & & \\
\hline \multicolumn{9}{|l|}{ Ethnicity ( $n \%$ ) } \\
\hline Caucasian & $168(89.4)$ & $154(88.5)$ & $14(100)$ & \multirow[t]{2}{*}{0.37} & - & \multirow[t]{2}{*}{-} & \multirow[t]{2}{*}{-} & \multirow[t]{2}{*}{ Caucasian } \\
\hline Other & $20(10.6)$ & $20(11.5)$ & $0(0)$ & & & & & \\
\hline \multicolumn{9}{|l|}{ Risk factor for HIV acquisition ( $n$ \%) } \\
\hline IDU & $20(11.4)$ & $18(11.0)$ & $2(15.4)$ & \multirow[t]{4}{*}{0.74} & 0.7 & \multirow[t]{4}{*}{$0.2-2.4$} & \multirow[t]{4}{*}{0.53} & \multirow[t]{4}{*}{ Sexual } \\
\hline Heterosexual intercourses & $85(47.7)$ & $77(47.0)$ & $8(61.5)$ & & 1 & & & \\
\hline Homo/bi-sexual intercourses & $71(40.3)$ & $68(41.4)$ & $3(23.1)$ & & & & & \\
\hline Transfusion & $1(0.6)$ & $1(0.6)$ & $0(0)$ & & & & & \\
\hline \multicolumn{9}{|l|}{ Physical activity at enrolment ( $n$ \%) } \\
\hline Yes & $58(30.8)$ & $57(32.8)$ & $1(7.1)$ & \multirow{2}{*}{$0.07^{\mathrm{b}}$} & 0.4 & \multirow[t]{2}{*}{$0.1-2.6$} & 0.08 & No \\
\hline No & $130(69.2)$ & $117(67.2)$ & $13(93.9)$ & & 1 & & & \\
\hline CD4 at enrolment ( $n \%)$ & & & & & & & & \\
\hline$<200$ & $34(18.1)$ & $32(18.4)$ & $2(14.3)$ & & 1 & & & $<200$ \\
\hline $200-500$ & $93(49.5)$ & $88(50.6)$ & $5(35.7)$ & $0.20^{\mathrm{c}}$ & 0.7 & $0.1-3.5$ & 0.64 & \\
\hline$>500$ & $61(32.4)$ & $54(31.0)$ & $7(50.0)$ & & 1.6 & $0.3-7.9$ & 0.55 & \\
\hline $\log _{10}$ HIV RNA at enrolment ( $n \%$ ) & & & & & & & & \\
\hline$\geq 5.00$ & $41(22.0)$ & $41(23.7)$ & $0(0)$ & $0.08^{\mathrm{b}}$ & - & - & - & $<5.00$ \\
\hline$<5.00$ & $145(78.0)$ & $132(76.3)$ & $13(100.0)$ & & & & & \\
\hline CD4 at last visit ( $n \%$ ) & & & & & & & & \\
\hline$<200$ & $10(5.4)$ & $9(5.2)$ & $1(7.1)$ & & 1 & & & \\
\hline $200-500$ & $83(44.4)$ & $76(43.9)$ & $7(50.0)$ & $0.54^{\mathrm{c}}$ & 1.2 & $0.1-11.5$ & 0.88 & \\
\hline$>500$ & $94(50.3)$ & $88(50.9)$ & $6(42.9)$ & & 0.8 & $0.1-8.0$ & 0.87 & \\
\hline Logistic & & & & & (OR) & & & \\
\hline$<200$ & & & & & & & & \\
\hline Detectable HIV RNA at last visit ${ }^{\mathrm{d}}$ & & & & & & & & \\
\hline Yes & $21(16.3)$ & $19(15.6)$ & $2(28.6)$ & $0.32^{\mathrm{b}}$ & 3.1 & $0.7-14.6$ & 0.15 & No \\
\hline No & $108(80.6)$ & $103(84.4)$ & $5(71.4)$ & & 1 & & & \\
\hline Logistic & & & & & $(\mathrm{OR})$ & & & \\
\hline Baseline FRS ( $n$ \%) & & & & & & & & \\
\hline$<5.00$ & $110(58.8)$ & $107(61.8)$ & $3(21.4)$ & & 1 & & & $<5.00$ \\
\hline $5.00-10.00$ & $42(22.5)$ & $38(22.0)$ & $4(28.6)$ & $0.0007^{\mathrm{c}}$ & 1.9 & $0.4-9.0$ & 0.39 & \\
\hline$\geq 10.00$ & $35(18.7)$ & $28(16.2)$ & $7(50.0)$ & & 7.5 & $1.8-31.2$ & 0.006 & \\
\hline FRS at last follow-up A (actual age & & & & & & & & \\
\hline$<5.00$ & $90(51.7)$ & $88(54.7)$ & $2(15.4)$ & & 1 & & & $<5.00$ \\
\hline $5.00-10.00$ & $41(23.6)$ & $38(23.6)$ & $3(23.1)$ & $0.0002^{\mathrm{c}}$ & 2.9 & $0.4-21.0$ & 0.28 & \\
\hline$\geq 10.00$ & $43(24.7)$ & $35(21.7)$ & $8(61.5)$ & & 9.3 & $1.6-53.5$ & 0.001 & \\
\hline Logistic & & & & & $(\mathrm{OR})$ & & & \\
\hline FRS at last follow-up B (baseline a & & & & & & & & \\
\hline$<5.00$ & $108(58.4)$ & $105(61.4)$ & $3(21.4)$ & & 1 & & & $<5.00$ \\
\hline $5.00-10.00$ & $36(19.5)$ & $34(19.9)$ & $2(14.3)$ & $0.001^{\mathrm{c}}$ & 1.5 & $0.2-10.5$ & 0.69 & \\
\hline$\geq 10.00$ & $41(22.2)$ & $32(18.7)$ & $9(64.3)$ & & 8.5 & $1.8-39.1$ & 0.006 & \\
\hline Logistic & & & & & (OR) & & & \\
\hline Positive criteria at baseline ( $n \%$ ) & & & & & & & & \\
\hline 0 & $62(33.0)$ & $62(35.6)$ & $0(0)$ & & - & - & - & 2 \\
\hline 1 & $76(40.4)$ & $70(40.2)$ & $6(42.9)$ & $0.001^{\mathrm{c}}$ & 0.4 & $0.1-1.3$ & 0.14 & \\
\hline 2 & $50(36.6)$ & $42(24.2)$ & $8(57.2)$ & & 1 & & & \\
\hline
\end{tabular}


Table 1 (Continued)

\begin{tabular}{|c|c|c|c|c|c|c|c|c|}
\hline Variable & Total & Without MS & With MS & $P$ & $\mathrm{RR}^{\mathrm{e}}$ & $95 \% \mathrm{Cl}^{\mathrm{e}}$ & $P^{e}$ & $\begin{array}{l}\text { Reference } \\
\text { variable }\end{array}$ \\
\hline Started at enrolment & $62(33.0)$ & $57(32.8)$ & $5(35.7)$ & \multirow{3}{*}{$0.16^{\mathrm{b}}$} & 0.4 & $0.1-1.5$ & 0.19 & \multirow[t]{3}{*}{ Naive } \\
\hline Started HAART during the study & $67(35.6)$ & $65(37.4)$ & $2(14.3)$ & & 0.4 & $0.1-2.1$ & 0.30 & \\
\hline Naive & $59(31.4)$ & $52(29.9)$ & $7(50.0)$ & & 1 & & & \\
\hline \multicolumn{9}{|l|}{ HAART (third agent) ( $n \%)$} \\
\hline NNRTI & $58(45.0)$ & $56(45.9)$ & $2(28.6)$ & $0.46^{\mathrm{b}}$ & 0.3 & $0.1-1.6$ & 0.14 & \multirow[t]{2}{*}{ Naive } \\
\hline PI & $60(46.5)$ & $56(45.9)$ & $4(57.1)$ & $0.70^{\mathrm{b}}$ & 0.7 & $0.2-2.5$ & 0.65 & \\
\hline
\end{tabular}

a Out of six menopausal women, two (33.3) had Metabolic Syndrome, versus one (2.6) out of 40 not menopausal (Fisher's exact test $P=0.04$ ).

b Fisher exact test.

c Mantel Haenzsel $\mathrm{Chi}^{2}$.

d Only patients on HAART.

e Adjusted for gender, age, BMI, HCV co-infection and physical activity (as appropriate). Sometimes the sums do not add up to 188 because of missing values.

Table 2

Changes from baseline during 3-year follow-up according to HAART.

\begin{tabular}{|c|c|c|c|c|c|c|c|c|c|}
\hline & \multicolumn{3}{|c|}{ Total $(n=188)$} & \multicolumn{2}{|c|}{ Naive $(n=59)$} & \multicolumn{2}{|c|}{ HAART $(n=129)$} & \multirow[t]{2}{*}{$P$} & \multirow[t]{2}{*}{$P^{\mathrm{a}}$} \\
\hline & Median & & IQ range & Median & IQ range & Median & IQ range & & \\
\hline SBP & 0.0 & & $-10.0-10.3$ & 0.0 & $-10.0-10.5$ & 0.0 & $-10.0-10.0$ & 0.91 & 0.36 \\
\hline DBP & 0.0 & & $-5.0-7.5$ & 0.0 & $-7.5-8.0$ & 0.0 & $-5.0-5.0$ & 0.87 & 0.39 \\
\hline Waist circumference & 1.0 & & $-1.7-5.0$ & 0.0 & $-2.0-4.0$ & 1.0 & $-1.0-7.0$ & 0.06 & 0.18 \\
\hline HDL cholesterol & 0.0 & & $-9.0-11.0$ & -6.0 & $-12.0-0.0$ & 4.0 & $-5.0-12.0$ & $<0.0001$ & $<0.0001$ \\
\hline Triglycerides & 8.5 & & $-20.0-54.0$ & 1.0 & $-32.0-24.0$ & 12.0 & $-15.0-58.0$ & 0.02 & 0.02 \\
\hline Blood glucose & 4.0 & & $-3.0-11.0$ & 1.0 & $-6.0-8.0$ & 6.0 & $-2.0-11.0$ & 0.03 & 0.01 \\
\hline FRS A (actual age) & 0.98 & & $-0.10-2.77$ & 1.08 & $0.06-2.45$ & 0.94 & $-0.11-2.80$ & 0.95 & 0.94 \\
\hline FRS B (baseline age) & 0.33 & & $-0.45-1.64$ & 0.40 & $-0.44-1.62$ & 0.31 & $-0.46-1.65$ & 0.91 & 0.92 \\
\hline MS criteria & & $N$ & $\%$ & $N$ & $\%$ & $N$ & $\%$ & $P$ & $P^{\mathrm{a}}$ \\
\hline $\mathrm{BP}$ & & 53 & 28.2 & 16 & 27.1 & 37 & 28.7 & 0.83 & 0.95 \\
\hline Waist circumference & & 25 & 13.3 & 10 & 17.0 & 15 & 11.6 & 0.32 & 0.89 \\
\hline HDL cholesterol & & 73 & 38.8 & 26 & 44.1 & 47 & 36.4 & 0.31 & 0.18 \\
\hline Triglycerides & & 71 & 37.8 & 14 & 23.7 & 57 & 44.2 & 0.007 & 0.008 \\
\hline Blood glucose & & 13 & 6.9 & 3 & 5.1 & 10 & 7.8 & 0.50 & 0.22 \\
\hline \multicolumn{10}{|c|}{ Number of positive criteria } \\
\hline 0 & & 45 & 23.9 & 16 & 27.1 & 29 & 22.5 & & \\
\hline 1 & & 70 & 37.2 & 27 & 45.8 & 43 & 33.3 & & \\
\hline 2 & & 59 & 31.4 & 9 & 15.2 & 50 & 38.8 & & \\
\hline 3 & & 10 & 5.3 & 5 & 8.5 & 5 & 3.9 & 0.44 & 0.41 \\
\hline $4-5$ & & 4 & 2.1 & 2 & 3.4 & 2 & 1.6 & & \\
\hline
\end{tabular}

a Adjusted for gender, age, BMI, HCV co-infection and initial level of variable (as appropriate).

significantly associated with MS; age more than 50 years was a significant determinant for MS development $(\mathrm{RR}=3.4,95 \% \mathrm{CI} 1.2-$ 11.0, $P=0.02$ ), whereas physical activity seemed protective, but not significantly (RR $=0.4,95 \% \mathrm{CI} 0.1-2.6, P=0.08)$.

Baseline FRS more than or equal to 10 was predictive of MS development $(\mathrm{RR}=7.5,95 \% \mathrm{CI} 1.8-31.2, P=0.006)$; FRS more than or equal to 10 at last follow-up was also associated to MS (OR).

HAART initiation did not appear significantly related to MS development, though the $R R=0.3$ suggested a protective role of NNRTIs.

During follow-up, the median FRS increase was 0.98; patients who developed MS had a higher FRS at baseline and were subjected to a higher median increase (6.0) in comparison to patients without MS (0.9). This difference also emerged when using the age at enrolment in the FRS equation (2.6 vs. 0.3 ) and suggested that factors other than ageing were responsible of FRS increase.

\subsection{Metabolic Syndrome criteria analysis}

As a secondary objective, we analyzed the single features of MS (Table 2) according to HAART initiation. Patients who remained naïve had baseline frequency of low HDL-C level significantly lower than patients who initiated HAART $(22.0 \%$ vs. $40.3 \%$,
$P<0.05)$. The baseline prevalence of other MS features were similar between groups. At study end (or at MS diagnose) HDL-C frequency did not differ, whereas TGL $>150 \mathrm{mg} / \mathrm{dL}$ were significantly more frequent in HAART patients.

During the observation period, these criteria may have varied either way, since BP, WC, HDL-C, TGL and BG could either increase or diminish (Table2).Toaccount for initial values, we analyzed the absolute changes in BP,WC, HDL-C, TGLand BG. A positive value indicate an increase and a negative value a decrease as compared to the baseline value.

HAART was associated to HDL-C: patients that started therapy during the study period showed a median HDL-C increase significantly higher than patients who remained treatment-naïve. Baseline CD4 cell count $<200$ cells $/ \mathrm{mm}^{3}$ was also related to HDL-C change: the lower the baseline CD4 cell count, the higher the HDL$C$ increase during the study period. This relation was mediated by the HAART initiation, being subjects with CD4 cell count less than 200 more likely to start HAART.

HAART duration was directly correlated with several metabolic changes (data not shown in tables): WC $(r=0.15, P=0.04)$, HDL-C $(r=0.26, P=0.0003)$. BG was also correlated $(r=0.11)$ but not significantly. Conversely, study time while still naïve was inversely correlated to HDL-C $(r=-0.20, P=0.007)$, WC $(r=-0.22, P=0.003)$ and BG $(r=-0.16, P=0.03)$. 
Table 3

Changes from baseline during 3-year follow-up according to HAART class.

\begin{tabular}{|c|c|c|c|c|c|c|c|c|c|}
\hline & \multicolumn{3}{|c|}{ Naive $(n=59)$} & \multicolumn{2}{|c|}{ NNRTI $(n=58)$} & \multicolumn{2}{|c|}{ PI $(n=60)$} & \multirow[t]{2}{*}{$P$} & \multirow[t]{2}{*}{$P^{\mathrm{e}}$} \\
\hline & Median & & IQ range & Median & IQ range & Median & IQ range & & \\
\hline SBP & 0.0 & & $-10.0-10.5$ & -3.3 & $-10.0-7.0$ & 0.0 & $-6.0-12.5$ & 0.28 & 0.12 \\
\hline DBP & 0.0 & & $-7.5-8.0$ & 0.0 & $-5.0-5.0$ & 0.5 & $-5.0-6.5$ & 0.69 & 0.22 \\
\hline Waist circumference & 0.0 & & $-2.0-4.0$ & 1.0 & $-2.0-5.0$ & 3.0 & $0.0-9.0$ & 0.0009 & $0.03^{\mathrm{a}}$ \\
\hline HDL cholesterol & -6.0 & & $-12.0-0.0$ & 6.5 & $-3.0-12.0$ & 4.0 & $-5.0-14.0$ & $<0.0001$ & $<0.0001^{\mathrm{b}}$ \\
\hline Triglycerides & 1.0 & & $-32.0-24.0$ & 5.5 & $-16.0-39.0$ & 34.5 & $-7.5-66.5$ & 0.01 & $0.001^{\mathrm{a}}$ \\
\hline Blood glucose & 1.0 & & $-6.0-8.0$ & 4.0 & $-1.0-11.0$ & 7.0 & $-3.5-13.0$ & 0.05 & $0.04^{\mathrm{b}}$ \\
\hline FRS A (actual age) & 1.08 & & $0.06-2.45$ & 0.37 & $-0.43-2.22$ & 1.15 & $0.38-4.31$ & 0.14 & 0.16 \\
\hline FRS B (baseline age) & 0.40 & & $-0.44-1.62$ & 0.07 & $-1.08-1.16$ & 0.65 & $-0.05-1.96$ & 0.12 & 0.14 \\
\hline MS criteria & & $\mathrm{N}$ & $\%$ & $\mathrm{~N}$ & $\%$ & $\mathrm{~N}$ & $\%$ & $P$ & $P^{e}$ \\
\hline $\mathrm{BP}$ & & 16 & 27.1 & 18 & 31.0 & 16 & 26.7 & 0.85 & 0.48 \\
\hline Waist circumference & & 10 & 17.0 & 6 & 10.3 & 9 & 15.0 & 0.57 & 0.55 \\
\hline HDL cholesterol & & 26 & 44.1 & 16 & 27.6 & 24 & 40.0 & 0.16 & $0.04^{\mathrm{c}}$ \\
\hline Triglycerides & & 14 & 23.7 & 19 & 32.8 & 31 & 51.7 & 0.005 & $0.10^{d}$ \\
\hline Blood glucose & & 3 & 5.1 & 6 & 10.3 & 3 & 5.0 & 0.42 & 0.09 \\
\hline Number of positive criteria & & & & & & & & 0.79 & 0.70 \\
\hline 0 & & 16 & 27.1 & 17 & 29.3 & 11 & 18.3 & & \\
\hline 1 & & 27 & 45.8 & 20 & 34.5 & 20 & 33.3 & & \\
\hline 2 & & 9 & 15.2 & 19 & 32.8 & 25 & 41.7 & & \\
\hline 3 & & 5 & 8.5 & 1 & 1.7 & 3 & 5.0 & & \\
\hline $4-5$ & & 2 & 3.4 & 1 & 1.7 & 1 & 1.7 & & \\
\hline
\end{tabular}

${ }^{a}$ NNRTI and naïve groups were significantly different from PI group ( $P=0.03$ and $P=0.01$ respectively).

b NNRTI and PI groups were significantly different from naïve group $(P<0.0001$ and $P=0.0003$ respectively).

c NNRTI group was significantly different from naïve group $(P=0.04)$.

d PI group was significantly different from naïve group $(P=0.01)$.

e Adjusted for gender, age, BMI, HCV co-infection and initial level of variable (as appropriate).

\subsection{Regimen analysis}

In Table 3, naïve patients were compared to those who started a PI-based or NNRTI-based therapy. Concerning the WC and the TGL changes from baseline, NNRTI treated and naïve patients were different from PI-treated ones that showed a significantly greater WC and TGL increase. However, compared to naïve patients, NNRTI treated and PI-treated subjects showed a significantly higher HDL$\mathrm{C}$ and BG increase. In the analysis of MS criteria, low HDL-C criterion was less frequent in the NNRTI group and TGL criterion was more frequent in the PI group (both significant if compared to naïve patients).

\section{Discussion}

The main findings of this study was the low MS incidence and the different trend of metabolic changes seen in HAART groups.

Other studies have prospectically followed patients to estimate MS incidence $[3,4,12]$. Compared to our results, Wand et al. [4] found a lower baseline prevalence (8.5\% with ATP-III MS definition vs. $12.3 \%$ ) and a higher incidence over a 3 -year observation period (12/100 patient-years vs. 2.6/100 patient-years). Another study [3] found a incidence of MS (15 cases/100 person-years) similar to Wand, [4] with a baseline prevalence of $24 \%$, but this study included both naïve and HAART-treated subjects, thus a direct comparison with our results was not feasible.

Conversely, Palacios et al. [12] surveyed a sample of naïve patients starting HAART at enrolment: MS at baseline was $16.6 \%$, and $25 \%$ after 48 weeks. During follow-up, 7/50 patients developed MS, leading an incidence of $14 / 100$ patients/year. Despite the similar baseline prevalence, MS incidence in our cohort was lower. However, our study was performed more recently, when new drugs were available and the physician alert on MS and obesity was higher compared to the past. This aspect may partially explain the low MS incidence observed.
We also focused on the comparison between patients who started HAART and the group that remained naïve. For each component of MS there is a complex interaction between HIV, therapy and central obesity. Some retrospective studies found a higher prevalence of MS associated with HAART use [3,13-16] and in particular with PIs, $[2,3,14,16]$ whereas others did not find any association $[4,12,17,18]$. Some authors suggested that HAART specifically affected the triglycerides levels $[5,19,20]$ and the insuline resistance [13]. Specific antiretroviral therapies are known to affect individual components of MS adversely, such as increasing triglycerides as lopinavir/ritonavir, [21,22] but also efavirenz [23]. Along this line, we found that the most frequent MS feature among patients starting HAART was TGL $>150 \mathrm{mg} / \mathrm{dL}$; the change from baseline was significantly higher in patients on HAART as compared to naïve subjects. NNRTI-based regimen seemed associated to lower TGL increase than PI-based regimens. Among subjects on PIs, lopinavir/ritonavir-treated patients had blood lipid levels similar to those on other PIs (data not shown in tables).

In our sample, PIs seemed related to a significant WC increase, as is well documented [24]. Moreover, use of indinavir and lopinavir/ritonavir has been associated with increasing fasting glucose $[25,26]$. In our study, we found a slight median increase of BG level, as well as a higher incidence of this MS component among patients on HAART; the former was statistically significant. Comparing PI- and NNRTI-based treatments with naïve patients, we found that both regimens were associated with a significant BG increase, though this feature did not emerge in the criteria analysis due to the low number of cases.

On the other hand, some therapies have been associated with improvements in metabolic profiles, such as increases in HDL cholesterol $[27,28]$. The association between HIV infection and low HDL is well established [29,30]. A recent study [31] suggested that HIV-associated dyslipidemia was, partially at least, related to chronic inflammation, and that the effect of HAART on HDL changes could be mediated by suppressing inflammation. In fact, inflammation induces major changes in HDL levels and composition. 
Interestingly, HDL levels and functions are altered in several immune-mediated disorders, such as rheumatoid arthritis, systemic lupus eritematosus, Crohn's disease and multiple sclerosis as well as during inflammatory responses. Changes in HDL levels may represent a predictable response to attenuation of inflammation [32]. Specifically, HIV infection and chronic inflammation impairs the first step of reverse cholesterol transport, namely cholesterol efflux from macrophages that plays a key role in maintaining cell cholesterol homeostasis [33].

In our sample, we found a significant HDL-C change from baseline, between naïve and treated patients, that led to a frequency of the feature higher in the former, though not significantly. In the regimen analysis, the frequency of HDL-C criteria was significantly lower in the NNRTI-based treatment, in comparison to PI and naïve subjects.

We also investigated the cardiovascular risk factors at baseline and at follow-up. The estimated cardiovascular risk at baseline and at the end of follow-up was unchanged comparing naïve and HAART-treated patients, suggesting that the FRS increase due to TGL and total cholesterol was possibly balanced by the concomitant HDL-C increase. In the light of this finding, HAART did not seem responsible for significant additional vascular damage.

The main limitation of this study was the low number of new MS cases that prevented us from finding potentially significant risk factors. In some cases, as in the analysis for drugs, the lack of statistical significance may be due to the lack of power.

However, this study has several strength. First of all, we have had the possibility to compare MS incidence in patients who started HAART, using the group that remained naïve as comparison. Then, we reliably collected information about potential confounders at baseline. Lastly, the 3-year follow-up period was adequate for our purpose.

In conclusion, we found a low MS incidence in our population of HIV-positive subjects, naïve at enrolment. Furthermore, the incidence of MS was similar in patients who remained naive and in those who started treatment. However, the features of MS were different in the two groups: in this sample, TGL and BG increases were more frequent in HAART-treated (in particular PItreated) patients and HDL-C decrease was more frequent in naive subjects, possibly as an expression of inflammatory responses. These characteristics suggest that, in untreated and treated patients, MS developed through different metabolic pathways.

\section{Disclosure of interest}

The authors declare that they have no conflicts of interest concerning this article.

\section{References}

[1] Ford ES. Risks for all-cause mortality, cardiovascular disease, and diabetes associated with the metabolic syndrome: a summary of the evidence. Diabetes Care 2005;28:1769-78.

[2] Jericò C, Knobel H, Montero M, et al. Metabolic Syndrome among HIV-infected patients: prevalence, characteristics, and related factors. Diabetes Care 2005;28:132-7.

[3] Jacobson DL, Tang AM, Spiegelman D, et al. Incidence of MS in a cohort of HIVinfected adults and prevalence relative to the US population. J Acquir Immune Defic Syndr 2006;43:458-66.

[4] Wand H, Calmy A, Carey DL, et al. INITIO Trial International Coordinating Committee. Metabolic syndrome, cardiovascular disease and type 2 diabetes mellitus after initiation of antiretroviral therapy in HIV infection. AIDS 2007;21:2445-53.

[5] Mondy K, Overton ET, Grubb J, et al. Metabolic syndrome in HIV-infected patients from an urban. Midwestern US outpatient population. Clin Infect Dis 2007;44:726-34.

[6] Mangili A, Jacobson DL, Gerrior J, Polak JF, Gorbach SL, Wanke CA. Metabolic syndrome and sub clinical atherosclerosis in patients infected with HIV. Clin Infect Dis 2007;44:1368-74.

[7] Worm SW, Lundgren JD. The Metabolic Syndrome in HIV. Best Pract Res Clin Endocrinol Metab 2011;25:479-86.
[8] De Socio GV, Parruti G, Quirino T, et al. For the CISAI study group. Identifying HIV patients with an unfavorable cardiovascular risk profile in the clinical practice: results from the SIMONE study. J Infect 2008;57:33-40.

[9] Bonfanti P, De Socio GVL, Marconi P, et al. Is metabolic syndrome associated to HIV infection per se? Results from the HERMES study. Curr HIV Res 2010;8(2):165-71.

[10] National Cholesterol Education Program (NCEP) Expert Panel on Detection, Evaluation, and Treatment of High Blood Cholesterol in Adults (Adult Treatment Panel III). Third Report of the National Cholesterol Education Program (NCEP) Expert Panel on Detection, Evaluation, and Treatment of High Blood Cholesterol in Adults (Adult Treatment Panel III) final report. Circulation 2002; 106: 3143-421.

[11] De Socio GVL, Martinelli C, Ricci E, et al. For the HERMES study group. Relations between cardiovascular risk estimates and sub clinical atherosclerosis in naive HIV patients: results from the HERMES Study. Int J STD AIDS 2010;21:267-72.

[12] Palacios R, Santos J, González M, Ruiz J, Márquez M. Incidence prevalence of the metabolic syndrome in a cohort of naïve HIV-infected patients: prospective analysis at 48 weeks of highly active antiretroviral therapy. Int J STD AIDS 2007;18:184-7.

[13] Bergersen BM, Schumacher A, Sandvik L, Bruun JN, Birkeland K. Important differences in components of the metabolic syndrome between HIV patients with and without highly active antiretroviral therapy, and healthy controls Scand J Infect Dis 2006:38:682-9.

[14] Samaras K, Wand H, Law M, et al. Prevalence of metabolic syndrome in HIVinfected patients receiving highly active antiretroviral therapy using International Diabetes Foundation and Adult Treatment Panel III criteria: associations with insulin resistance, disturbed body fat compartmentalization, elevated C reactive protein, and corrected hypoadiponectinemia. Diab Care 2007;30:113-9.

[15] Falasca K, Ucciferri C, Manzoli L, et al. Metabolic syndrome and cardiovascular risk in HIV-infected patients with lipodystrophy. Int J Immunopathol Pharmacol 2007;20:519-27.

[16] Jevtovic DJ, Dragovic G, Salemovic D, Ranin J, Djurkovic-Djakovic O. The metabolic syndrome, an epidemic among HIV-infected patients on HAART. Biomed Pharmacother 2009;63:337-42.

[17] Bonfanti P, Giannattasio C, Ricci E, et al. HIV and Metabolic Syndrome: a comparison with the general population. J Acquir Immune Defic Syndr 2007;45:426-31

[18] Sobieszczyk ME, Hoover DR, Anastos K, et al. Prevalence and predictors of metabolic syndrome among HIV-Infected and HIV-Uninfected women in the Women's Interagency HIV study. J AIDS 2008;48:272-80.

[19] Baum MK, Rafie C, Lai S, et al. Coronary heart disease (CHD) risk factors and metabolic syndrome in HIV-positive drug users in Miami. Am J Infect Dis 2006;2:173-9.

[20] Hansen BR, Petersen J, Haugaard SB, et al. The prevalence of metabolic syndrome in Danish patients with HIV infection: the effect of antiretroviral therapy. HIV Medications 2009;10:378-87.

[21] Fontas E, van Leth F, Sabin CA, et al. Lipid profiles in HIV-infected patients receiving combination antiretroviral therapy: are different antiretroviral drugs associated with different lipid profiles? J Infect Diseases 2004; 189:1056-74.

[22] Leon A, Martinez E, Sarasa M, et al. Impact of steady-state lopinavir plasma levels on plasma lipids and body composition after 24 weeks of lopinavir/ ritonavir-containing therapy free of thymidine analogues. JAntimicr Chemother 2007:60:824-30.

[23] van Leth F, Phanuphak P, Stroes E, et al. Nevirapine and efavirenz elicit different changes in lipid profiles in anti-retroviral therapy-naive patients infected with HIV-1. PLoS Medicine 2004:1:19.

[24] Carr A, Samaras K, Chisholm DJ, Cooper DA. Pathogenesis of HIV-1-protease inhibitor-associated peripheral lipodystrophy, hyperlipidaemia, and insulin resistance. Lancet 1998;351:1881-3.

[25] Noor MA, Lo JC, Mulligan K, et al. Metabolic effects of indinavir in healthy HIVseronegative men. Acquired Immune Deficiency Syndrome 2001;15:F11-8.

[26] Lee GA, Seneviratne T, Noor MA, et al. The metabolic effects of lopinavir/ ritonavir in HIV-negative men. Acquired Immune Deficiency Syndrome 2004;18:641-9.

[27] Franssen R, Sankatsing RR, Hassink E, et al. Nevirapine increases high-density lipoprotein cholesterol concentration by stimulation of apolipoprotein A-I production. Arterioscler Thromb Vasc Biol 2009;29:1336-41.

[28] Phillips AN, Carr A, Neuhaus J, et al. Interruption of antiretroviral therapy and risk of cardiovascular disease in persons with HIV-1 infection: exploratory analyses from the SMART trial. Antiviral Therapy 2008;13:177-87.

[29] Riddler SA, Smit E, Cole SR, et al. Impact of HIV infection and HAART on serum lipids in men. JAMA 2003;289:2978-82.

[30] El-Sadr WM, Mullin CM, Carr A, et al. Effects of HIV disease on lipid, glucose and insulin levels: results from a large antiretroviral-naïve cohort. HIV Med 2005;6:114-21.

[31] Baker JV, Neuhaus J, Duprez D, et al. Inflammation predicts changes in highdensity lipoprotein particles and apolipoprotein A1 following initiation of antiretroviral therapy. AIDS 2011;25:2133-42.

[32] Norata GD, Pirillo A, Ammirati E, Catapano AL. Emerging role of high density lipoproteins as a player in the immune system. Atherosclerosis 2011. doi: 10.1016/i.atherosclerosis.2011.06.045.

[33] Mujawar Z, Rose H, Morrow MP, Pushkarsky T, Dubrovsky L, Mukhamedova N, et al. Human immunodeficiency virus impairs reverse cholesterol transport from macrophages. PLoS Biol 2006;4:e365. 